\title{
Síndrome de Doege Potter
}

\section{Doege-Potter syndrome}

\author{
Héctor Fabio Sandoval-Alzate, July Marcela Parra-Gamboa, Andrea Angulo-Casalis \\ - Bogotá, D.C. (Colombia)
}

DOI: https://doi.org/10.36104/amc.2020.1503

\begin{abstract}
Resumen
El síndrome de Doege Potter es un síndrome caracterizado por hipoglucemia severa asociada a tumor fibroso de la pleura. Presentamos el caso de una paciente de 67 años con antecedente por biopsia de tumor fibroso de pleura, quien seis meses después de este diagnóstico presenta episodios de alteración del estado de conciencia con desorientación y documentación de hipoglucemia con triada de Whipple presente. Se realiza test de ayuno el cual es positivo para hipoglucemia no hiperinsulinémica y dado sus antecedentes, se hace el diagnóstico de un síndrome de Doege Potter. Se realiza manejo quirúrgico con resección total de masa tumoral con posterior resolución de la hipoglucemia. (Acta Med Colomb 2020; 45: 189-192. DOI: https://doi.org/10.36104/amc.2020.1503).

Palabras clave: hipoglucemia, neoplasias pleurales, factor de crecimiento similar a la insulina tipo II.
\end{abstract}

Dr. Héctor Fabio Sandoval-Alzate: Médico Internista-Endocrinólogo. Profesor Facultad de Medicina Universidad Nacional de Colombia. Profesor Escuela de Medicina y Ciencias de la Salud Universidad del Rosario; Dra. July Marcela Parra-Gamboa: Médico General, Universidad del Rosario; Dra. Andrea AnguloCasalis: Médico General, Universidad del Rosario. Hospital Universitario Mayor-Méderi. Bogotá, D.C. (Colombia).

Correspondencia: Dr. Héctor Fabio SandovalAlzate. Bogotá, D.C. (Colombia).

E-mail : hecfasan@yahoo.com

Recibido: 16/IX/2019 Aceptado: 13/IV/2020

\section{Introducción}

La hipoglucemia en el paciente no diabético se define como una glucemia menor a $55 \mathrm{mg} / \mathrm{dL}$ (1), considerándose como una emergencia médica y cuyos síntomas son diversos y dependerán fundamentalmente de la severidad de ésta, clasificándose de manera usual como síntomas neuroglucopénicos (cambios en comportamiento, somnolencia, confusión, convulsiones y coma), síntomas autonómicos adrenérgicos (temblor, palpitaciones, ansiedad) y síntomas autonómicos colinérgicos (hambre, diaforesis, parestesias) (2). Dentro de las causas descritas como origen de la hipoglucemia en el paciente no diabético, se caracterizan las de origen farmacológico, como lo son las producidas por las sulfonilúreas, cibenzolamina, antibióticos como pentamidina, quinina y el alcohol; déficit de hormonas como glucagón, cortisol, epinefrina; falta de aporte de glucosa por inanición o desnutrición; pacientes críticos con falla hepática, cardiaca o renal; hiperinsulinismo endógeno por nesidioblastosis, postcirugía de bypass gástrico o por tumores neuroendocrinos pancreáticos funcionales como el insulinoma. Así mismo, la hipoglucemia no hiperinsulinémica es producida por neoplasias no pancreáticas como el fibrosarcoma, liposarcoma, rabdomiosarcoma, leucemia, linfoma, teratoma, tumores fibrosos (solitarios o múltiples) y el mesotelioma $(3,4)$.

El tumor fibroso solitario de pleura es una neoplasia poco común. Este tumor cursa en su mayoría de forma benigna representando el $8 \%$ de los tumores benignos en tórax y $10 \%$ de los tumores pleurales. Se localiza en $30 \%$ en la pleura visceral o parietal y de forma extratorácica predominantemente en el peritoneo, pericardio y mediastino $(5,6)$. Este tumor recibe otros nombres como mesotelioma localizado, mesotelioma fibroso benigno o fibroma benigno localizado (5). Dado que en su gran mayoría son de comportamiento benigno y de lento crecimiento, pueden pasar desapercibidos 
por largo tiempo de manera asintomática. Sin embargo, $10 \%$ de estos tumores fibrosos pueden presentar un comportamiento maligno con recurrencia local o metastásica, contemplándose dentro de las variables de malignidad la presencia de una alta celularidad, una actividad mitótica de cuatro o más mitosis/10 campos de alto poder y necrosis (7-9). En 2013 se describió un sistema de puntuación de factores de riesgo para predecir un comportamiento agresivo, con un grado significativo de recurrencia si se presentan al menos tres de los seis criterios siguientes: localización en la pleura parietal, un tamaño igual o mayor a $10 \mathrm{~cm}$, hipercelularidad, atipia nuclear, cuatro o más mitosis/10 campos de alto poder y la presencia de necrosis $(10,11)$.

En los pacientes con tumor fibroso solitario de pleura, la hipoglucemia ocurre en menos de 5\% de los casos (5). La hipoglucemia refractaria se ha asociado más frecuentemente cuando estos tumores se originan en el peritoneo o cuando alcanzan un tamaño "gigante" en la pleura (tamaños superiores a los $10 \mathrm{~cm})(6)$. Al tener una asociación de hipoglucemia junto al tumor solitario de pleura, se presenta un síndrome paraneoplásico referido en la literatura como el síndrome de Doege Potter.

El síndrome de Doege Potter fue descrito en 1930 como un síndrome caracterizado por hipoglucemia severa, sostenida y refractaria, asociada a tumores no pancreáticos, más específicamente a tumores fibrosos originados con mayor frecuencia en la pleura (12). En general es un síndrome que cursa con una presentación clínica inespecífica, con síntomas asociados al compromiso pulmonar: tos, disnea, dolor torácico, derrame pleural (6); a nivel metabólico con hipoglucemia, siendo este el principal criterio diagnóstico (12) y que, en su mecanismo fisiopatológico se caracteriza por presentar niveles bajos de insulina, péptido $\mathrm{C}$ indetectable, así como niveles normales de IGF-I (12). El tratamiento se fundamenta principalmente en la extirpación del tumor con fines curativos con la consecuente mejoría sintomática.

Se presenta el caso de una paciente con clínica inicialmente respiratoria con síntomas intratorácicos, documentación de una masa pleural con diagnóstico histológico por biopsia de tumor fibroso solitario de pleura y posteriormente aparición de síntomas asociados a hipoglucemia severa, con triada de Whipple presente: síntomas o signos de hipoglucemia, niveles bajos de glucemia y resolución de los síntomas después de su corrección (1). Adicionalmente se le practicó un test de ayuno de 72 horas con resultado positivo para hipoglucemia no hiperinsulinémica a las pocas horas de iniciado el test, considerándose así, el diagnóstico de un síndrome de Doege Potter.

\section{Reporte de caso}

Se trata de una mujer de 67 años que consulta por presentar cuadro clínico de seis semanas de evolución consistente en episodios de alteración del estado de conciencia dado por alucinaciones, desorientación, alteración del comportamien- to, desconexión con el medio, con posterior recuperación de éste y amnesia del cuadro. Seis meses previos a este episodio, la paciente había consultado por tos con esputo hemoptoico, donde se realiza tomografía computarizada (TC) de tórax contrastada con hallazgo de masa pleural de 17 x 12 x $9 \mathrm{~cm}$ sugestivo de tumor fibroso. Se le realizó biopsia de pleura por técnica trucut con reporte de tumor fibroso con inmunoreactividad difusa en las células tumorales con vimentina, CD99, BCL2 y CD34 con un índice de proliferación celular de 5\% medido con Ki67. Los marcadores P53, NSE, S100, AML y desmina son negativos. Dos meses posteriores a esta biopsia se realiza otra biopsia por técnica trucut de control la cual confirmó el tumor fibroso de pleura con tumor fusocelular sin atipia, sin necrosis ni actividad mitótica considerándose como un tumor benigno e inicia trámites para valoración e intervención por cirugía de tórax.

Por sus síntomas de ingreso se descarta evento cerebro vascular, síncope de origen neurogénico/cardiogénico con realización de una TC cerebral, doppler de vasos de cuello, electroencefalograma y resonancia magnética cerebral sin hallazgos relevantes, al igual que un ecocardiograma transtorácico con FEVI conservada. Dado cuadro clínico de etiología no claro, fue valorada por el servicio de psiquiatría y neuropsicología descartando cuadro de origen psicosomático.

Por desaturaciones durante los episodios descritos, se realizó nuevamente radiografía de tórax con reporte de opacidad basal izquierda de contorno superior bien definido (Figura 1), y realización de una nueva TC de tórax contrastada con reporte de una imagen compatible con tumor fibroso de pleura izquierdo, el cual ha incrementado de manera significativa sus dimensiones comparativamente con el control previo (abril 2018), granuloma calcificado de aspecto residual en lóbulo superior derecho (Figura 2).

Durante la preparación para la realización del estudio imagenológico, y aproximadamente luego de seis horas de ayuno, presenta alteración del estado de conciencia y diaforesis, por lo que se realiza glucometría con reporte de 41 $\mathrm{mg} / \mathrm{dL}$. Se administran 20 gr de dextrosa con glucometría de control en $62 \mathrm{mg} / \mathrm{dL}$. Durante las siguientes 24 horas presentó hipoglucemia sintomática refractaria a manejo a pesar de bolos de dextrosa, por lo que se dejó infusión continua con mejoría en su estado de conciencia, así como de sus glucometrías. Por hipoglucemia refractaria de causa no clara, se descartan otras patologías endocrinológicas como diabetes mellitus (hemoglobina glicosilada en 5.2\%), enfermedad tiroidea (TSH $1.8 \mathrm{IU} / \mathrm{L}$ ) e insuficiencia adrenal (cortisol basal de las 8 de la mañana en $221 \mathrm{nmol} / \mathrm{L}$ ).

Paciente con antecedente de tumor fibroso de pleura (diagnóstico histopatológico) asociado a hipoglucemia sintomática severa de difícil manejo con triada de Whipple presente, por lo que se consideró como diagnóstico presuntivo un síndrome paraneoplásico. Se practicó test de ayuno de 72 horas, con hipoglucemia confirmada en las primeras horas de la prueba, con reporte de péptido $\mathrm{C}$ 

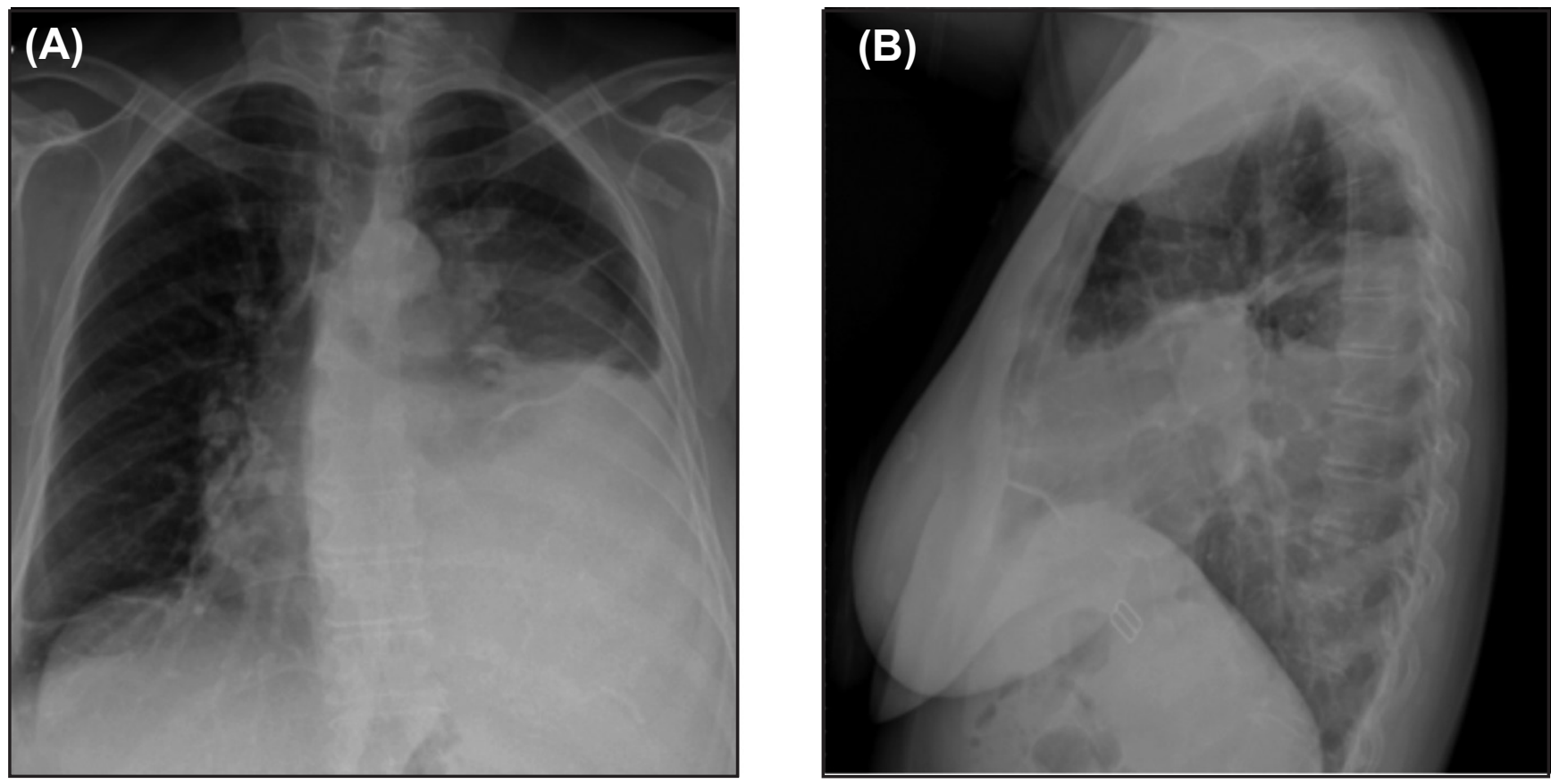

Figura 1. Radiografía de tórax: masa que ocupa la base pulmonar izquierda. A: postero-anterior. B: lateral.
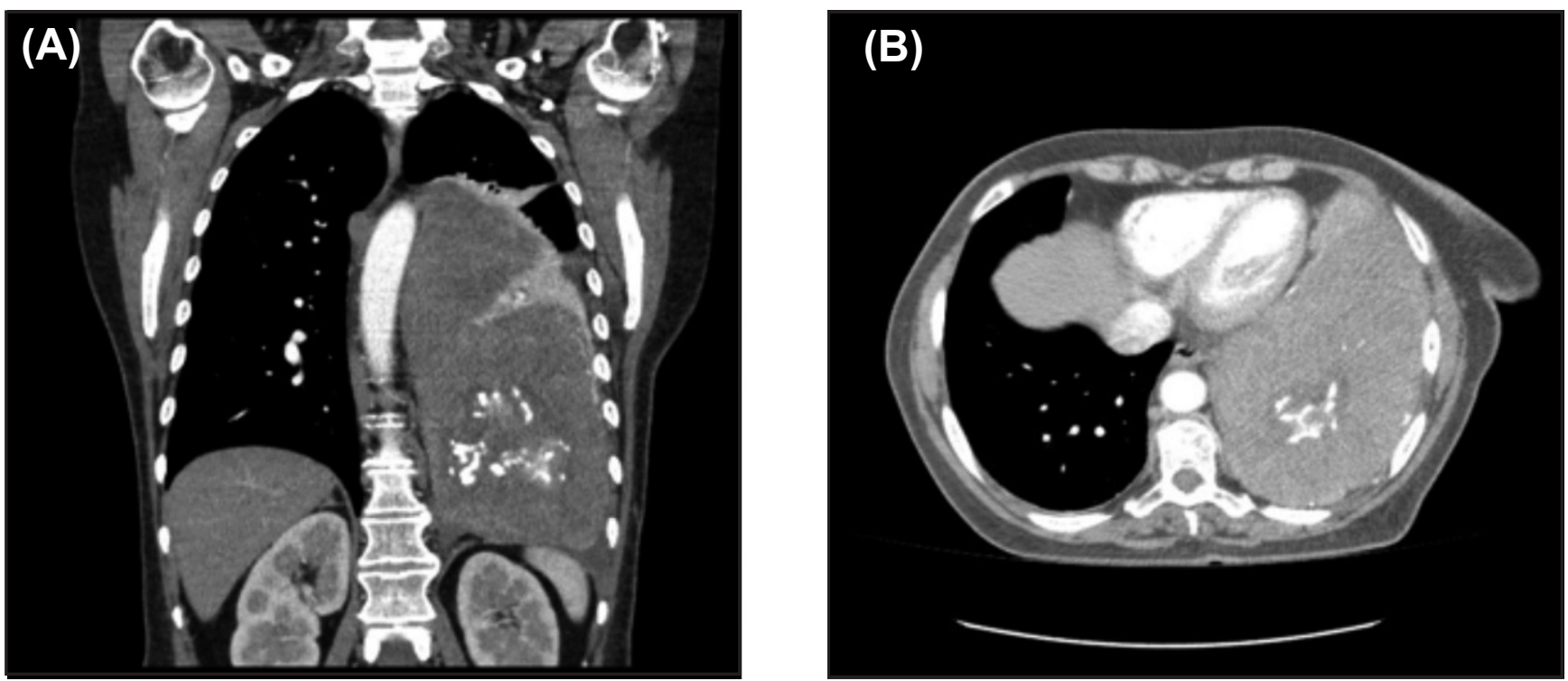

Figura 2. Tomografía contrastada de tórax: Masa extrapulmonar sólida con calcificaciones en su interior. A: corte coronal. B: corte transversal en ventana pulmonar.

menor a $33.1 \mathrm{pmol} / \mathrm{L}$ e insulina reportada menor a 4.2 $\mathrm{pmol} / \mathrm{L}$, teniendo así una paciente con un tumor fibroso de pleura con hipoglucemia no hiperinsulinémica severa sintomática, concluyéndose como primera posibilidad un diagnóstico de síndrome de Doege Potter. Se practicó manejo complementario con prednisolona a dosis de 15 mg cada 12 horas, con lo que se logró resolver transitoriamente las hipoglucemias, mientras era valorada por el servicio de cirugía de tórax y llevada a manejo quirúrgico para resección de tumor fibroso de pleura. Los hallazgos intraquirúrgicos demostraron un tumor de 15 x 10 x $10 \mathrm{~cm}$ de diámetro sólido en contacto con diafragma en el lóbulo inferior izquierdo.

En el postoperatorio la paciente presentó mejoría clínica, sin nuevos episodios de hipoglucemia ni alteración del estado de conciencia, sin requerimiento de aportes adicionales de glucosa tras suspensión de la prednisolona. Reporte de patología con evidencia de tumor fibroso de pleura de 17 × 15 × 9 cm, bordes de resección libres de tumor. En seguimiento ambulatorio a la paciente durante los siguientes meses, permaneció asintomática, sin nuevos episodios de hipoglucemia. 


\section{Discusión}

El síndrome de Doege Potter es causado principalmente por tumores intratorácicos hipoglucemiantes (12), en general por un tumor fibroso solitario, aunque algunos casos reportan la presentación como tumor múltiple (12). Se ha descrito como un síndrome paraneoplásico poco frecuente (13) con una incidencia de 2.8 en 100000 habitantes (11) y con una media de edad al diagnóstico entre la sexta y la octava década de la vida (13).

Los tumores pueden alcanzar un gran tamaño antes de ser sintomáticos, y cuando se presentan, hay síntomas intratorácicos como tos, disnea, derrame pleural o extratorácicos como la osteoartropatía hipertrófica o la hipoglucemia severa sostenida (13). En nuestro caso la paciente se encuentra dentro del grupo etario usual de presentación y se trató de un tumor fibroso solitario de pleura. Como criterio diagnóstico se tiene en cuenta el cuadro clínico producido por hipoglucemia sintomática severa secundaria a un mayor consumo de glucosa por parte del tumor $(5,6)$ y/o a la secreción del factor de crecimiento similar a la insulina tipo II (IGF-II), generando un estado de reducción en los niveles de insulina y un desplazamiento de la somatomedina $\mathrm{C}$ (IGF-I) de su proteína transportadora (6). En el caso presentado se documentó una hipoglucemia no hiperinsulinémica en el test de ayuno, en el escenario de una hipoglucemia severa persistente; sin embargo no se realizó medición sérica de IGF-II dado la no disponibilidad para su realización en nuestro medio.

Teniendo en cuenta que el principal síntoma del síndrome es la hipoglucemia, la cual es la causa de la mayoría de las complicaciones en estos pacientes, el tratamiento "curativo" es la extirpación quirúrgica del tumor (13). En cuanto al manejo sintomático en su mayoría, consiste en el uso de dextrosa intravenosa en bolo o en infusión según la respuesta clínica. Sin embargo, existen manejos complementarios vistos en la literatura, utilizándose terapias con glucagón, análogos de la somatostatina, hormona de crecimiento recombinante y por supuesto, glucocorticoides, siendo este último, el que nos permitió hacer un control de la hipoglucemia como terapia puente mientras se realizaba la extirpación quirúrgica del tumor. Los glucocorticoides y la hormona de crecimiento de forma combinada o en monoterapia, son los agentes más frecuentemente utilizados para el manejo sintomático, e incluso son una alternativa cuando el tumor tiene criterios de inoperabilidad. Se prefiere el uso de glucocorticoide dado su bajo costo y su efectividad, ya que disminuye directamente los niveles de IGF II (14). Otros manejos adicionales descritos cuando el tumor es irresecable, han sido la quimioterapia combinada de temozolomida y bevacizumab (13).

Dentro del diagnóstico histopatológico, estos tumores solitarios benignos de pleura suelen ser positivos para marcadores como vimentina y otros menos específicos como el CD34, bcl-2 o CD99 y negativo para citoqueratina (13).

A pesar de presentar un bajo índice de mortalidad (dado que la mayoría de estos tumores son de naturaleza benignos), puede tener un comportamiento similar a un tumor maligno ocasionando un crecimiento más agresivo o desarrollando síndromes paraneoplásicos.

Tener en cuenta este síndrome es de vital importancia ante un tumor fibroso solitario, ya que es la hipoglucemia la que puede terminar afectando de manera más severa, las condiciones de estos pacientes.

\section{Referencias}

1. Philip E. Cryer, Lloyd Axelrod, Ashley B. Grossman, Simon R. Heller, Victor M. Montori, Elizabeth R. Seaquist, F. John Service. Evaluation and Management of Adult Hypoglycemic Disorders: An Endocrine Society Clinical Practice Guideline, The Jour of Clin End \& Met. 2009; 94 (3):709-728.

2. Mathew P, Thoppil D. Hypoglycemia. StatPearls Publishing, 2018. Available from: https://www.ncbi.nlm.nih.gov/books/NBK534841/

3. Gomez A, Henao $\mathbf{C}$ et al. Relevancia del monitoreo continuo de glucosa en la práctica clínica: revisión de la evidencia. Rev Col de End, Diab y Metab. 2018; 5(3): $33-42$

4. Nicolau J, Gimenez M, Miro O. Hipoglucemia. JANO. 2006.

5. Cedeno B, Areosa A. Síndrome de Doege-Potter. A propósito de un caso. Rev Esp Geriatr Gerontol. 2018;53(5):299-305.

6. Santos S.M. Fernandez R. Canto H. Doege-Potter syndrome: What hypoglycemia hid. Rev Clin Esp. 2018.

7. England D, Hochholzer L et al. Localized benign and malignant fibrous tumors of the pleura. A clinicopathologic review of 223 cases. Am J Surg Pathol. 1989; 13 (8):640-650

8. Gold JS, Antonescu CR, Hadju C, Ferrone CR, Hussain M, Lewis JJ, et al. Clinicopathologic correlates of solitary fibrous tumors. Cancer 2002; 94:10571068

9. Scrimgeour LA, Grada Z, Aswad BI, et al. Lessons learned from an untreated "benign" thoracic tumor. Ann Thorac Surg 2017;103:e135-7.

10. Tapias, L.F., Mino-Kenudson, M., Lee, H. et al. Risk factor analysis for the recurrence of resected solitary fibrous tumours of the pleura: a 33-year experience and proposal for a scoring system. Eur J Cardiothorac Surg. 2013; 44: 111-117.

11. Chen S, Zheng Y, et all. A broad ligament solitary fibrous tumor with Doege-Potter síndrome. Wolt Kluwer journ Health. 2018; 93:39.

12. Cameselle R, Piñeiro $\mathbf{L}$, et al. Tumor endotoracico hipoglicemiante Sindrome de Doege Potter. Archivo de Bronconeumologia.1990; 26 (7): 317-319.

13. Ogunsakin A, Hilsenbeck H, et al. Recurrent Severe Hypoinsulinemic Hypoglycemia Responsive to Temozolomide and Bevacizumab in a Patient With Doege-Potter Syndrome. Am J Med Sci. 2018; 356(2):181-184.

14. Lujan M, Mejia S, et all. Síndrome de Doege-Potter Tumor fibroso de pleura irresecable asociado a hipoglucemia. Acta Med Colomb, 2009; 34 (4). 